Article

\title{
Exatecan Antibody Drug Conjugates Based on a Hydrophilic Polysarcosine Drug-Linker Platform
}

\author{
${\text { Louise Conilh }{ }^{1,2}(\mathbb{D} \text {, Guy Fournet }}^{3}{ }^{(0}$, Eric Fourmaux ${ }^{1,2}$, Angélique Murcia ${ }^{1,3}$, Eva-Laure Matera ${ }^{2}$, \\ Benoît Joseph ${ }^{3,+} \oplus$, Charles Dumontet ${ }^{2,4,+}$ and Warren Viricel ${ }^{1, *,+}+\mathbb{C}$ \\ 1 Mablink Bioscience, 69 rue de la république, 69002 Lyon, France; 1.conilh@mablink.com (L.C.); \\ e.fourmaux@mablink.com (E.F.); a.murcia@mablink.fr (A.M.) \\ 2 Centre de Recherche en Cancérologie de Lyon, INSERM 1052, CNRS 5286, Université de Lyon, \\ 69008 Lyon, France; eva-laure.matera@univ-lyon1.fr (E.-L.M.); charles.dumontet@chu-lyon.fr (C.D.) \\ 3 Institut de Chimie et Biochimie Moléculaires et Supramoléculaires, UMR CNRS 5246, Université de Lyon, \\ 69100 Villeurbanne, France; guy.fournet@univ-lyon1.fr (G.F.); benoit.joseph@univ-lyon1.fr (B.J.) \\ 4 Hospices Civils de Lyon, 69000 Lyon, France \\ * Correspondence: w.viricel@mablink.com \\ + These authors contributed equally.
}

Citation: Conilh, L.; Fournet, G.; Fourmaux, E.; Murcia, A.; Matera, E.-L.; Joseph, B.; Dumontet, C.; Viricel, W. Exatecan Antibody Drug Conjugates Based on a Hydrophilic Polysarcosine Drug-Linker Platform. Pharmaceuticals 2021, 14, 247.

https://doi.org/10.3390/ph14030247

Academic Editor: Mary J. Meegan

Received: 14 February 2021

Accepted: 6 March 2021

Published: 9 March 2021

Publisher's Note: MDPI stays neutral with regard to jurisdictional claims in published maps and institutional affiliations.

Copyright: (c) 2021 by the authors. Licensee MDPI, Basel, Switzerland. This article is an open access article distributed under the terms and conditions of the Creative Commons Attribution (CC BY) license (https:// creativecommons.org/licenses/by/ $4.0 /)$.

\begin{abstract}
We herein report the development and evaluation of a novel HER2-targeting antibody-drug conjugate (ADC) based on the topoisomerase I inhibitor payload exatecan, using our hydrophilic monodisperse polysarcosine (PSAR) drug-linker platform (PSARlink). In vitro and in vivo experiments were conducted in breast and gastric cancer models to characterize this original ADC and gain insight about the drug-linker structure-activity relationship. The inclusion of the PSAR hydrophobicity masking entity efficiently reduced the overall hydrophobicity of the conjugate and yielded an ADC sharing the same pharmacokinetic profile as the unconjugated antibody despite the high drug-load of the camptothecin-derived payload (drug-antibody ratio of 8). Tra-Exa-PSAR10 demonstrated strong anti-tumor activity at $1 \mathrm{mg} / \mathrm{kg}$ in an NCI-N87 xenograft model, outperforming the FDA-approved ADC DS-8201a (Enhertu), while being well tolerated in mice at a dose of $100 \mathrm{mg} / \mathrm{kg}$. In vitro experiments showed that this exatecan-based ADC demonstrated higher bystander killing effect than DS-8201a and overcame resistance to T-DM1 (Kadcyla) in preclinical HER2+ breast and esophageal models, suggesting potential activity in heterogeneous and resistant tumors. In summary, the polysarcosine-based hydrophobicity masking approach allowsfor the generation of highly conjugated exatecan-based ADCs having excellent physicochemical properties, an improved pharmacokinetic profile, and potent in vivo anti-tumor activity.
\end{abstract}

Keywords: antibody-drug conjugates; polysarcosine; deruxtecan; topoisomerase I inhibitor; camptothecin

\section{Introduction}

Antibody-drug conjugates (ADCs) are biotherapeutics that combine highly cytotoxic molecules with the targeting property of antibodies to specifically kill cancer cells [1]. These agents possess reduced systemic toxicity and aim to improve the narrow therapeutic window associated with conventional chemotherapies. One hundred years after the emergence of the Magic Bullet concept [2], 10 ADCs are now clinically approved, 5 of which were approved during the past two years [3-7]. More than 90 ADCs are currently under clinical evaluation [8,9]. The vast majority of approved ADCs as well as those currently in clinical trials deliver microtubule inhibitors (auristatins, maytansinoids) or DNA-alkylating agents (calicheamicin, pyrrolobenzodiazepines, duocarmycins) and are limited to a drugantibody ratio (DAR) of 2 to 4 . This moderate DAR value has until recently been considered to be optimal to obtain efficient ADCs achieving acceptable pharmacokinetic properties, in vivo efficacy, and safety. This is due to intrinsic hydrophobicity of the drug-linker, 
which negatively impacts antibody tertiary structure, causes increased plasma clearance, increases toxicity, and ultimately leads to a reduced therapeutic window [10-14]. In this context and because only $1-2 \%$ of the injected payload dose ultimately reach the tumor site [15], highly cytotoxic small molecules with $\mathrm{IC}_{50}$ 's in the pico- to low nano-molar range are therefore required to generate moderately conjugated yet potent ADCs $[16,17]$. This finding has greatly oriented the field towards ADCs based on very potent DNA alkylating agents (such as pyrrolobenzodiazepines) and site-specific DAR 2 bioconjugation technologies [18-20]. Several promising and strongly cytotoxic ADCs entered clinical trials but showed unsatisfactory results due to non-manageable undesirable off-target toxicities [21,22], suggesting that ADCs based on less potent but safer cytotoxic small molecules could benefit from a wider clinical therapeutic window.

Topoisomerase I (Topo I) inhibitors represent the most recent breakthrough in ADC payload innovation with the approval of two ADCs containing camptothecin (CPT) analogues: trastuzumab deruxtecan (DS-8201a-Enhertu ${ }^{\circledR}$ ) and sacituzumab govitecan (IMMU132-Trodelvy ${ }^{\mathrm{TM}}$ ) [23,24]. Topo I inhibitors trigger cell apoptosis through their specific binding at the DNA-topoisomerase interface, leading to the inhibition of DNA supercoiling and entanglement, resulting in DNA damage and cell death $[25,26]$. This class of payloads is mainly composed of CPT analogues, wherein irinotecan and topotecan are FDA-approved chemotherapeutics [27,28]. Topo I inhibitors are 10- to 100-fold less potent than microtubule-targeting and DNA-alkylating agents, which largely explains the lack of initial interest for these payloads in first generation ADCs. However the recent development of carefully designed hydrophilic drug-linkers able to overcome payload hydrophobicity in order to generate highly-conjugated ADCs [29-35] allows us to reconsider Topo I inhibitors as potential ADC payloads. This is illustrated by the recent FDA approval of trastuzumab deruxtecan for the treatment of unresectable or metastatic HER2+ breast cancer and later in 2021 for the treatment of HER2+ locally advanced or metastatic gastric cancer. This ADC is composed of the HER2-targeting monoclonal antibody ( $\mathrm{mAb}$ ) trastuzumab, homogeneously attached to eight molecules of deruxtecan, a drug-linker based on the active topo I inhibitor agent DXd and a novel GGFG quadripeptide-based cleavable moiety [23]. Preclinical evaluations and clinical trials demonstrated a favorable pharmacokinetic profile, excellent anti-tumor activity and safety profiles, as well as a strong bystander killing effect, surpassing the previously approved trastuzumab emtansine (T-DM1-Kadcyla ${ }^{\circledR}$ ) in several studies [23,36-41].

We have previously reported a hydrophilic monodisperse polysarcosine-based druglinker platform (PSARlink ${ }^{\mathrm{TM}}$ ) that demonstrated significant reduction in ADC hydrophobicity level in spite of high DAR 8 MMAE conjugation, associated with improved physicochemical and pharmacological properties [29]. In the present work, we report the translation of our ADC platform to the topoisomerase I inhibitor compound exatecan. This active agent presents promising potential but its use as an ADC payload has been limited because of its hydrophobicity and challenging biophysical properties, which appear to be strongly caused by the steric hindrance around the stereo-defined primary amine [42]. Exatecan is a partially water-soluble and non-prodrug derivative of CPT [43] that is closely related but not identical to DXd (Figure S1). The conjugation of exatecan to trastuzumab through the glucuronidase-cleavable polysarcosine-based linker reported therein (Exa-PSAR10) ultimately provides (i) a homogeneous DAR 8 conjugation, (ii) an improved hydrophilic profile of the resulting ADC, (iii) an exquisite drug-linker plasma stability and favorable pharmacokinetic profile, (iv) a potent in vivo activity against breast and gastric cancer models, and (v) a highly potent in vitro bystander killing effect as well as a strong cytotoxicity against T-DM1-resistant cells.

\section{Results}

\subsection{Drug-Linker Conception, Bioconjugation, and Physicochemical Characterization of ADCs}

Following known chemical procedures [29], synthesis of the Exa-PSAR10 drug-linker was conducted (Figure 1 and Supplementary Materials). This enzyme-cleavable drug-linker 
includes a $\beta$-glucuronidase-sensitive trigger unit [44] to allow intracellular release of the final cytotoxic agent exatecan after endocytosis of the ADC [45]. To maximize drug-linker hydrophilicity, we included an orthogonal monodisperse PSAR unit as an hydrophobicity masking entity [29]. As we knew that a PSAR length of 12 sarcosine residues was an optimal value for the monomethyl auristatin E (MMAE) payload [29] and that exatecan is slightly less hydrophobic than MMAE $\left(\log P_{\text {exatecan }}=1.67-3.29\right.$ and $\log \mathrm{P}_{\mathrm{MMAE}}=3.44-4.61$ as predicted in silico [46]), we selected a length of 10 sarcosine residues. Finally, an autohydrolyzable aryl-maleimide bioconjugation head [47] was selected to prevent premature and deleterious in vivo deconjugation of the drug-linker by retro-Michael rearrangement with albumin in plasma [48]. A drug-linker Exa-PSAR0 lacking the PSAR hydrophobicity masking unit was also synthesized and was used as a negative control.
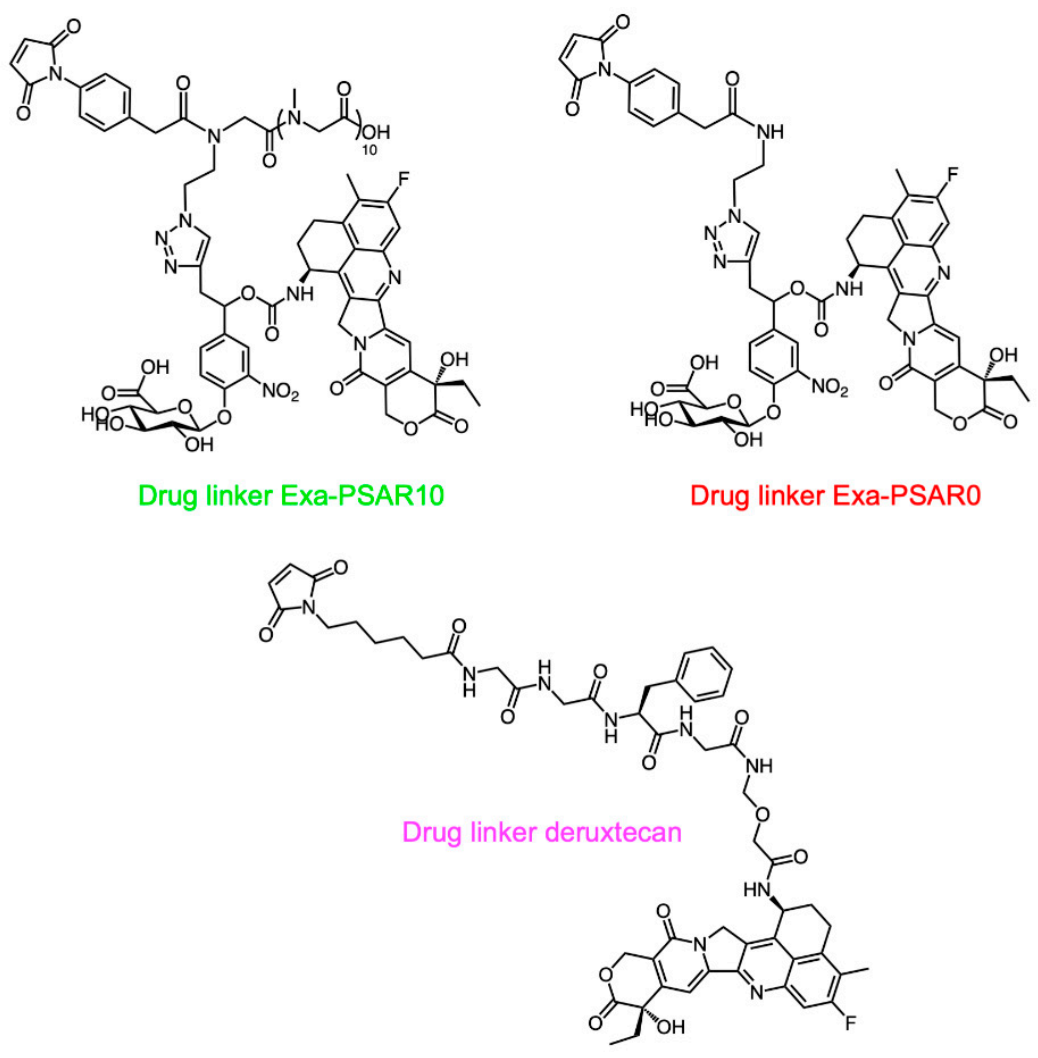

Figure 1. Chemical structures of the antibody-drug conjugates (ADC) drug-linkers used in the present study. See Supplementary Materials for detailed chemical synthetic procedures and exact ADC structures.

Drug-linkers were conjugated to the native inter-chain cysteines of reduced trastuzumab following a straightforward bioconjugation protocol in order to obtain homogeneous DAR 8 ADCs (Figure S2). Bioconjugation yields were above 80\% for Tra-Exa-PSAR10 and Tra-Exa-PSAR0 ADCs, and around 60-70\% for Tra-deruxtecan (DS-8201a linker). Analytical characterization by denaturing reversed phase chromatography-mass spectrometry (RPLC-MS) confirmed efficient conjugation and homogeneity of the conjugates (Figure S3).

Hydrophobic interaction chromatography (HIC) profiles of the conjugates showed that the two ADCs based on the glucuronide-exatecan modality (Tra-Exa-PSAR10 and Tra-ExaPSAR0) appeared almost as hydrophilic as the native antibody, despite the grafting of eight exatecan payloads per antibody (Figure 2A). No significant differences in HIC retention time were observed between the PSAR10 ADC and the PSAR0 negative control ADC. This result was not in accordance with our previous work with MMAE payload [29], where the negative control ADC lacking the hydrophobicity masking entity (PSAR0) showed a very significant increase in HIC retention time. The Tra-deruxtecan ADC eluted later in the HIC 
chromatogram, indicating a higher hydrophobicity level compared to the Exa-PSAR10 and Exa-PSAR0 conjugates. Size exclusion chromatography (SEC) chromatograms indicated that all ADCs were $95 \%+$ monomeric and that no aggregation occurred during formulation (Figure 2B). Ex vivo rat plasma stability studies by immunocapture followed by RPLCMS were also conducted (Figure 2C). Tra-Exa-PSAR10 and Tra-Exa-PSAR0 were found to be stable during the 7-day incubation period, as no premature glucuronide-cleavage was observed as well as no maleimide deconjugation. This could be expected as these two drug-linkers incorporate a stabilized auto-hydrolyzable maleimide bioconjugation head [47]. It was observed that Tra-deruxtecan lost approximately $20-40 \%$ of its cargo by maleimide deconjugation during the first days of incubation. This result is expected as the maleimidocaproyl bioconjugation headgroup that is used in the deruxtecan and vedotin (mc-vc-PAB-MMAE) drug-linkers is known to be susceptible to such retro-Michael deconjugations $[48,49]$.

A

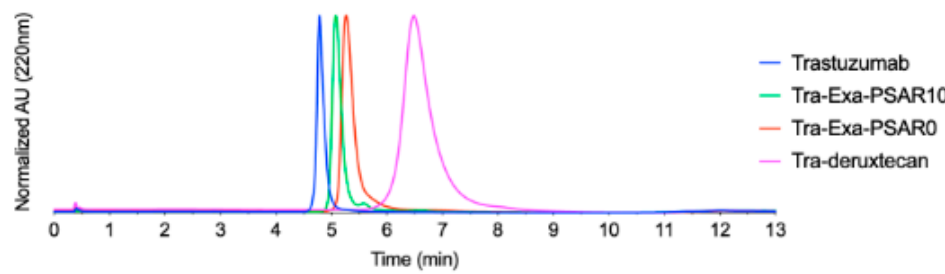

C

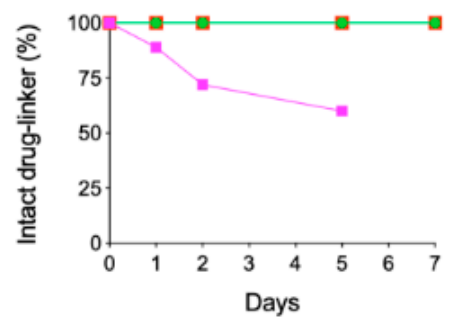

B

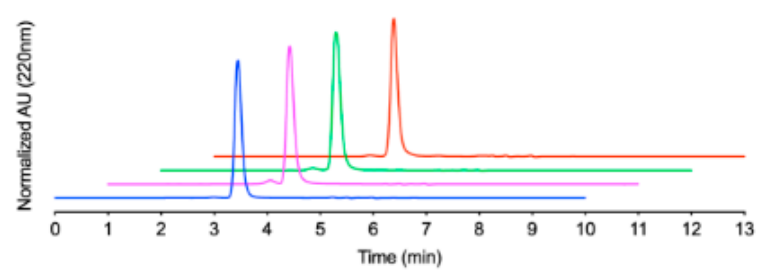

D

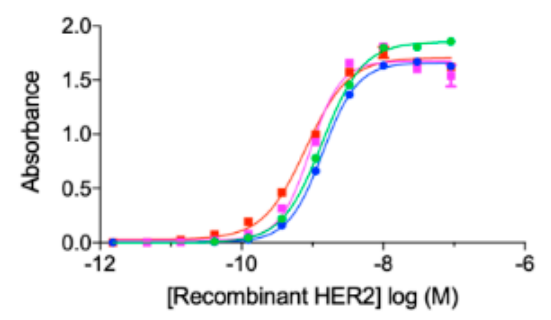

Figure 2. Physicochemical characterization of ADCs. (A) Hydrophobic Interaction Chromatograms (HIC) of DAR8 ADCs. (B) Size Exclusion Chromatograms (SEC) of DAR8 ADCs. (C) Ex-vivo rat plasma stability studies, as assayed by immunocapture and reversed phase HPLC-mass spectrometry. Low immunocapture recovery of the heavy-chain of the Tra-deruxtecan conjugate prevented us to report stability data at day 7 for this ADC. (D) HER2 ELISA binding affinity profiles of ADCs.

\subsection{DAR8 Conjugation and Orthogonal Polysarcosine Inclusion Did Not Negatively Impact Binding to HER2}

Antigen binding of unconjugated trastuzumab and trastuzumab conjugated either to Exa-PSAR10, Exa-PSAR0, or deruxtecan drug-linkers was assessed by ELISA (Figure 2D). No antigen-binding alteration of trastuzumab was observed when the antibody was fully reduced and conjugated at DAR 8 with each of the three drug-linkers, as $\log (\mathrm{EC} 50)$ values were $-8.9,-8.9,-9.0$, and -9.1 . In vitro cell binding was also assessed by flow cytometry using APC-labelled versions of the unconjugated and conjugated trastuzumab in HER2-positive cell lines (Figure 3A). Similar level of binding of the four tested entities was observed in two HER2-positive cell lines, SKBR-3 and MDA-MB-453, as compared to the isotype control. These results suggest that neither DAR 8 conjugation nor orthogonal incorporation of the polysarcosine hydrophobicity masking entity negatively impacted the antibody quaternary structure or masked its complementarity-determining regions (CDR). 
A

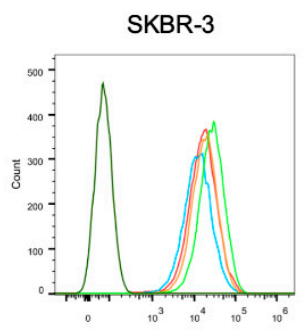

APC: HER-2

B
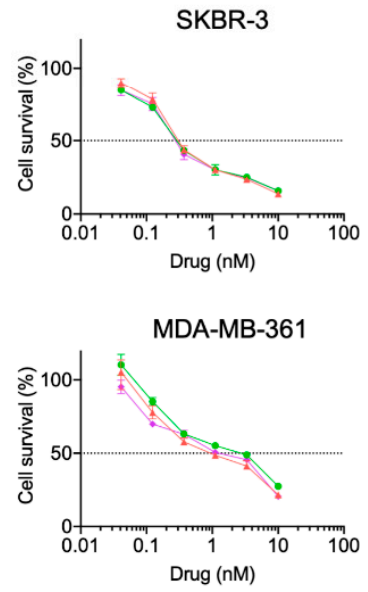

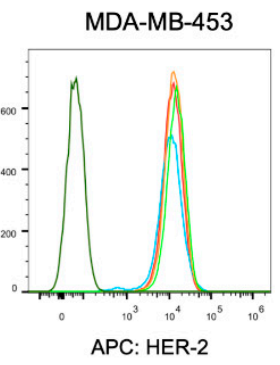

$$
\begin{array}{ll}
\square & \text { Control isotype } \\
\square & \text { Tra-Exa-PSAR10 } \\
\square & \text { Tra-Exa-PSAR0 } \\
\square & \text { Tra-deruxtecan } \\
\square & \text { Trastuzumab }
\end{array}
$$

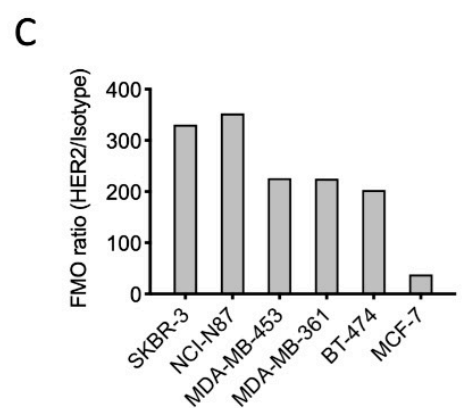

MDA-MB-453
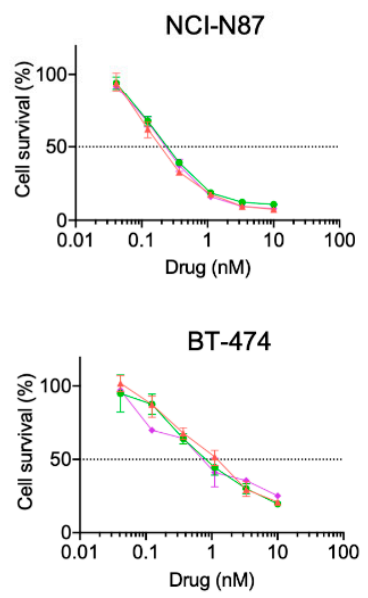

- Tra-Exa-PSAR10

^Tra-Exa-PSARO

- Tra-deruxtecan

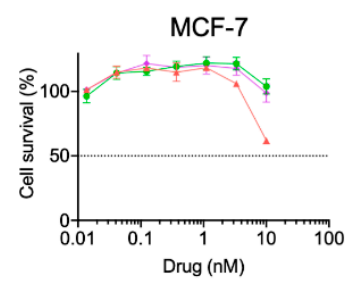

Figure 3. In vitro evaluation of Tra-Exa-PSAR10. (A) Trastuzumab and ADC cell binding assayed by flow cytometry. (B) In vitro cytotoxicity of ADCs in breast and gastric HER2+ and HER2- (MCF-7) cancer cell lines after 6-day exposure to trastuzumab conjugates, as assayed by MTT assay, $n=3$. (C) HER2 cell surface expression in breast and gastric tumor cell lines as assayed by flow cytometry.

\subsection{Tra-Exa-PSAR10 ADC Displayed Strong In Vitro Cytotoxicity in HER2-Positive Cell Lines}

In vitro activity of Tra-Exa-PSAR10 was assessed by in vitro cytotoxicity assay and compared to Tra-Exa-PSAR0 and Tra-deruxtecan (Figure 3B). The three ADCs presented equal sub-nanomolar $\mathrm{IC}_{50}$ 's in the five HER2-expressing cell lines (Figure $3 \mathrm{C}$ ), i.e., SKBR-3 $\left(\mathrm{IC}_{50}=0.18 \pm 0.04 \mathrm{nM}\right), \mathrm{NCI}-\mathrm{N} 87\left(\mathrm{IC}_{50}=0.20 \pm 0.05 \mathrm{nM}\right), \mathrm{MDA}-\mathrm{MB}-453\left(\mathrm{IC}_{50}=0.20 \pm 0.10 \mathrm{nM}\right)$, MDA-MB-361 $\left(\mathrm{IC}_{50}=2.0 \pm 0.8 \mathrm{nM}\right)$, and BT-474 $\left(\mathrm{IC}_{50}=0.9 \pm 0.4 \mathrm{nM}\right)$, and no cytotoxicity against MCF-7 ( $\mathrm{IC}_{50}>10 \mathrm{nM}$ ), a HER-2 negative breast cancer cell line. These results are in accordance with previously reported in vitro cytotoxicity data with DS-8201a on SKBR-3 and NCI-N87 cell lines with $\mathrm{IC}_{50} \mathrm{~s}$ of 0.05 and $0.17 \mathrm{nM}$, respectively [23]. This series of experiments suggest that highly conjugated exatecan-based ADCs display strong in vitro cytotoxicity against breast and gastric cancer cells, comparable to that of the FDA-approved ADC trastuzumab-deruxtecan (DS-8201a).

2.4. Tra-Exa-PSAR10 Showed a Favorable PK Profile and Strong In Vivo Activity in HER2+Breast and Gastric Cancer Models

We first assessed the total ADC pharmacokinetic (PK) profile of Tra-Exa-PSAR10 in Sprague-Dawley rats using anti-human IgG ELISA, and compared it to unconjugated trastuzumab, Tra-Exa-PSAR0, and Tra-deruxtecan. A dose of $3 \mathrm{mg} / \mathrm{kg}$ was selected on the basis of previous knowledge with DAR8 ADCs $[29,30]$ and to be above saturable dose range in order to obtain linear and predictable clearance profiles [50]. As previously observed with highly conjugated antibodies [13,29], trastuzumab conjugated to eight molecules of exatecan (Tra-Exa-PSAR0) and exhibited an unfavorable accelerated plasma clearance (Figure 4A and Figure S4). The excessive hydrophobicity of highly conjugated ADCs has been shown to be the major factor of a poor pharmacokinetic profile [12]. The orthogonal inclusion of the PSAR10 hydrophobicity masking entity to the drug-linker 
structure successfully restored the same PK profile as the native unconjugated trastuzumab. Interestingly, the PK profile of Tra-deruxtecan also mimicked that of trastuzumab, despite the fact that this ADC was found to be somewhat hydrophobic by HIC (Figure 2A).

A

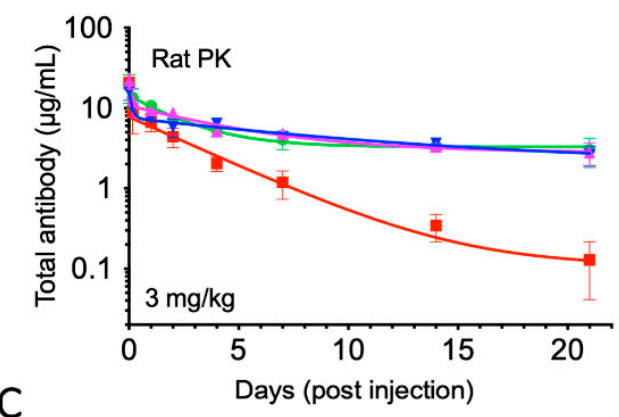

C

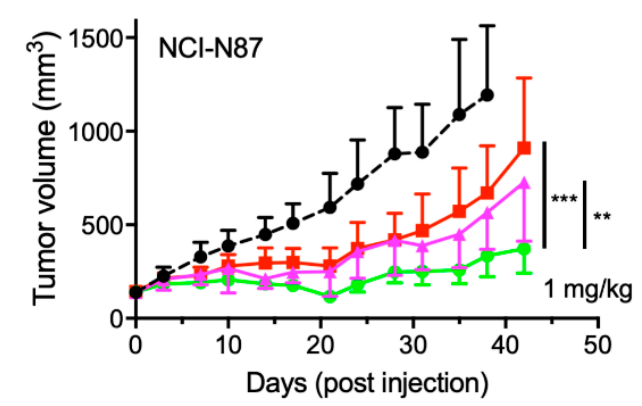

B
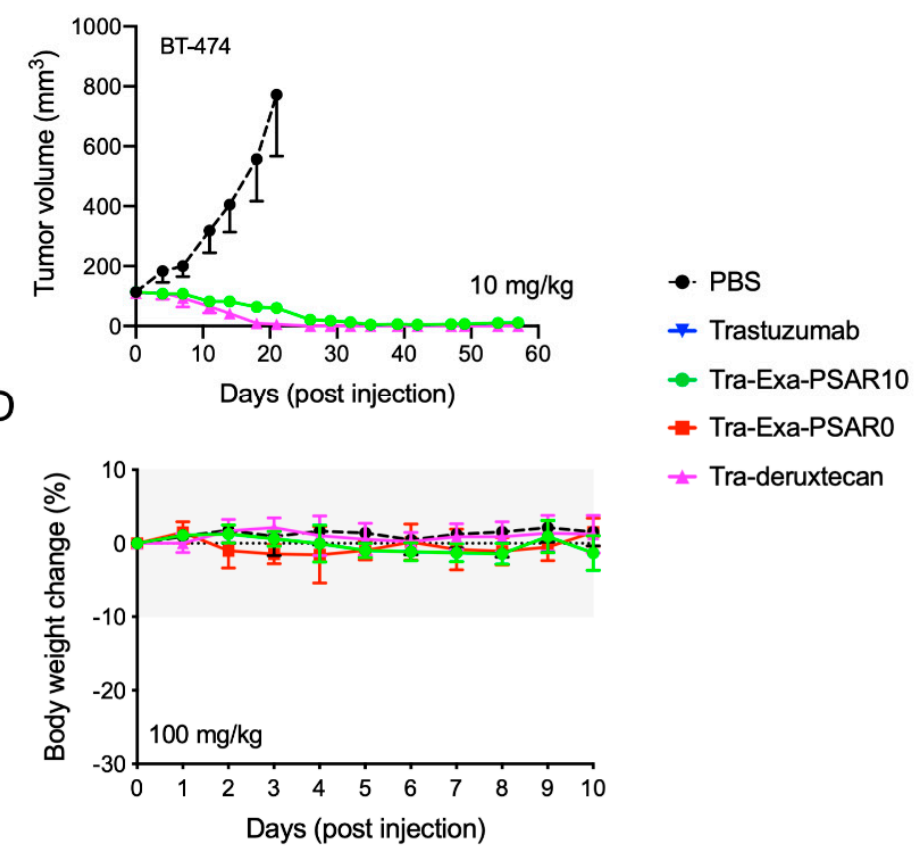

Figure 4. In vivo evaluation of Tra-Exa-PSAR10. (A) ADC pharmacokinetic study in Sprague-Dawley rats after a single intravenous ADC dose of $3 \mathrm{mg} / \mathrm{kg}$. Total ADC concentration was assayed by anti-human IgG ELISA. (B) Antitumor activity in HER2+ SCID/BT-474 breast cancer model following a single intravenous ADC dose of $10 \mathrm{mg} / \mathrm{kg}$. (C) Antitumor activity in HER2+ SCID/NCI-N87 gastric cancer model following a single sub-curative intravenous ADC dose of $1 \mathrm{mg} / \mathrm{kg}$. (D) Mice tolerability experiment following a single intraperitoneal ADC dose of $100 \mathrm{mg} / \mathrm{kg} .\left(^{* *}: p<0.01{ }^{* * *}: p<0.001\right)$.

As a first preliminary in vivo efficacy experiment, Tra-Exa-PSAR10 and Tra-deruxtecan were evaluated in the HER2+ breast cancer model BT-474 (Figure 4B). At a single exploratory ADC dose of $10 \mathrm{mg} / \mathrm{kg}$, a complete and prolonged remission of the tumors was observed in all groups. To further explore potential discrepancies in activity between the three drug-linkers of the study, we used the HER2+ gastric cancer model NCI-N87 at a sub-curative ADC dose of $1 \mathrm{mg} / \mathrm{kg}$ (Figure 4C). This dose was selected considering a previously reported NCI-N87 xenograft study with DS-8201a [37]. The tumor growth rate was significantly reduced in all groups compared to the control group, albeit to different extents. The most potent anti-tumor activity was observed with Tra-Exa-PSAR10, which was significantly more efficacious than its PSAR-lacking counterpart Tra-Exa-PSAR0 and the DXd-based ADC Tra-deruxtecan (Figure S5). These results suggest that the favorable PK profile enabled by the presence of the PSAR hydrophobicity masking entity translates into an improved in vivo efficacy. A first exploratory tolerability assessment was then conducted in SCID mice for these three ADCs (Figure 4D). No body weight changes, signs of distress, or inability to drink or eat were observed in animals for up to 10 days after an intraperitoneal injection of the ADCs at $100 \mathrm{mg} / \mathrm{kg}$. These results suggest a good systemic tolerance for the three topoisomerase I inhibitor-based ADCs.

\subsection{Tra-Exa-PSAR10 Displayed a Strong Bystander Activity In Vitro}

We first assessed the passive membrane diffusion of exatecan and compared its permeability coefficient (Pe) to DXd's using a parallel artificial membrane permeability (PAMPA) assay (Figure 5A). Exatecan exhibited a higher Pe value compared to DXd $\left(4.2 \times 10^{-6}\right.$ and $3.0 \times 10^{-6} \mathrm{~cm} / \mathrm{s}$, respectively) suggesting a better ability to passively cross lipid cell 
membranes. We then investigated the bystander killing potential of Tra-Exa-PSAR10 using an in vitro culture system. Single cell lines and co-cultures of HER2- (A549) and HER2+ (SKBR-3) cell lines were performed at different ratios and exposed to either $10 \mathrm{nM}$ of T-DM1 (negative control), DS-8201a, or Tra-Exa-PSAR10. After five days of incubation, cells were sorted for HER2 expression by flow cytometry. As expected, monocultured A549 cells displayed very modest to no sensitivity to the three ADCs at $10 \mathrm{nM}$, whereas the monocultured SKBR-3 cells were highly sensitive (Figure 5B,C). Co-culture experiments using various ratios of A549/SKBR3 cells showed that the HER2- A549 cells were killed when the co-cultures were exposed to DS-8201a and Tra-Exa-PSAR10 but not when exposed to T-DM1. Remarkably this effect was more pronounced for Tra-Exa-PSAR10 than for DS-8201a, especially at the 1:1;1:2, and 1:4 cell ratios (HER2-A549/HER2+SKBR-3). This observation could be explained by the stronger passive membrane permeability of exatecan compared to that of DXd.

A

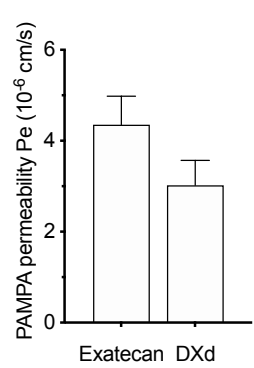

C
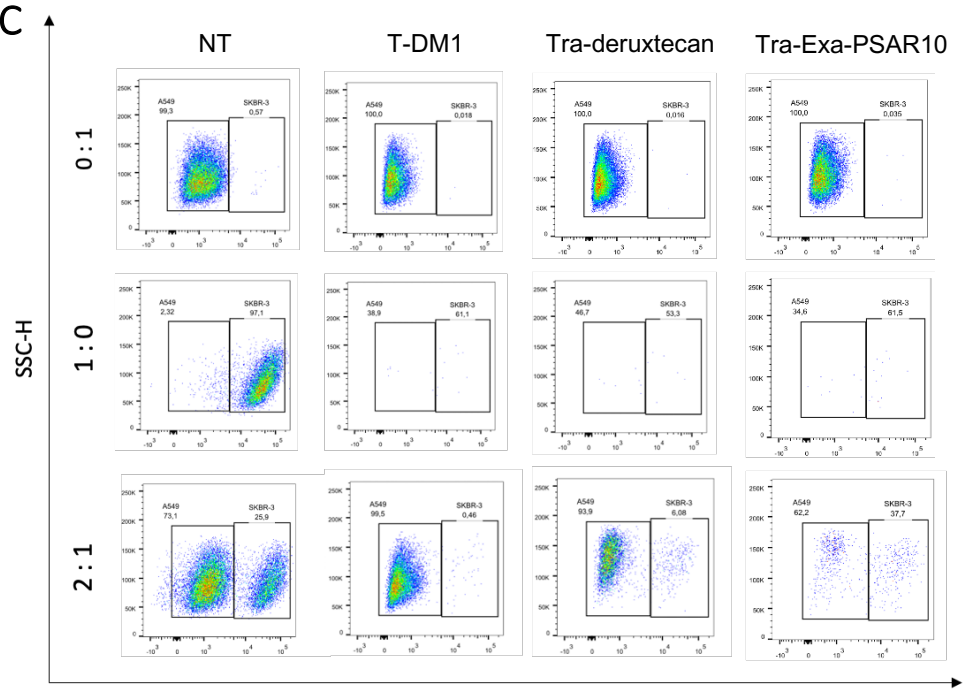

APC: HER-2
SKBR-3 (HER2+) A549 (HER2-)
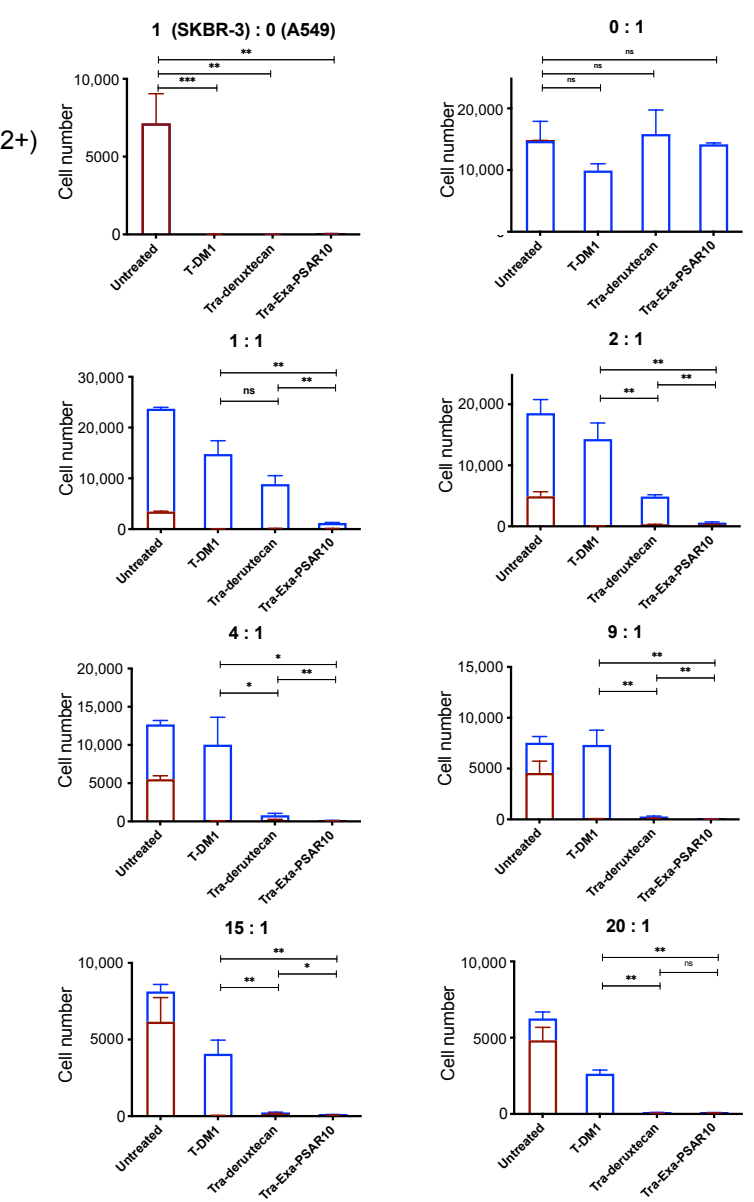

Figure 5. Bystander killing effect of Tra-Exa-PSAR10 compared to T-DM1 and trastuzumab-deruxtecan (DS-8201a) in co-culture in vitro. (A) Passive cell membrane diffusion of Exatecan and DXd payloads as assayed by a Corning ${ }^{\circledR}$ Gentest $^{\mathrm{TM}}$ PAMPA assay. (B) In vitro bystander killing effect of Tra-Exa-PSAR10 compared to Tra-deruxtecan in co-cultured SKBR-3 (HER2+) and A549 (HER2-) cells that were treated with $10 \mathrm{nM}$ ADCs for 5 days. Cell number and ratio of HER2+ and HER2- cells were determined by flow cytometry. T-DM1 (Kadcyla ${ }^{\circledR}$ ) was used as a negative control. (C) Representative flow cytometry data presented for 0:1, 1:0 and 2:1 cell ratio, showing no impact of the ADCs on HER2- A549 cells (0:1), activity against HER2+ SKBR-3 cells (1:0) and a stronger bystander activity of Tra-Exa-PSAR10 compared to Tra-deruxtecan. ns: not significant, $\left.{ }^{*}: p<0.05,{ }^{* *}: p<0.01,{ }^{* * *}: p<0.001\right) . n=3$.

\subsection{Tra-Exa-PSAR10 Overcame T-DM1 Resistance in Breast Cancer Models In Vitro}

T-DM1 (trastuzumab emtansine- - Kadcyla $^{\circledR}$ )-resistant cell lines were generated in our laboratory in HER2+esophageal OE-19 and HER2+ breast MDA-MB-361 cell lines, either in 
the absence (TR) or presence of ciclosporin A (TCR) [51,52]. This phenotype was confirmed using an in vitro cytotoxicity assay (Figure 6A). In MDA-MB-361 TR and TCR cell lines, $\mathrm{IC}_{50}$ 's of T-DM1 were increased 21- and 7-fold, respectively, compared to the parental cell line. In OE-19 TR and TCR cell lines, $\mathrm{IC}_{50}$ 's of T-DM1 were significantly increased sixfold compared to the parental OE-19-sensitive cell line.

A

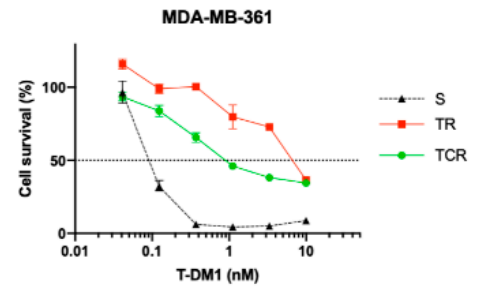

B

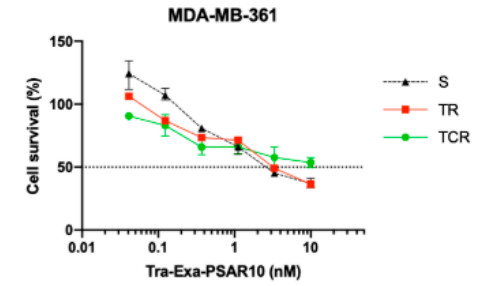

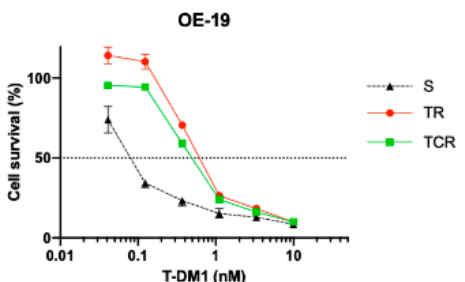

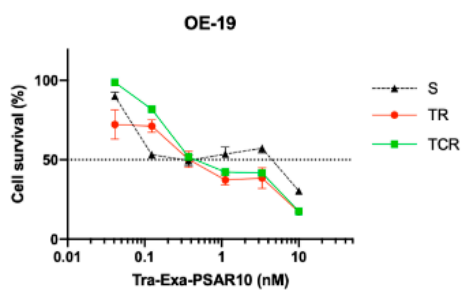

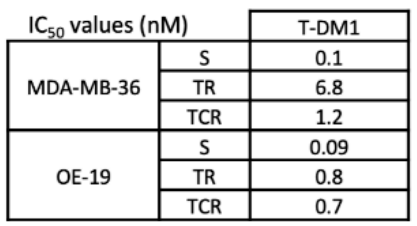

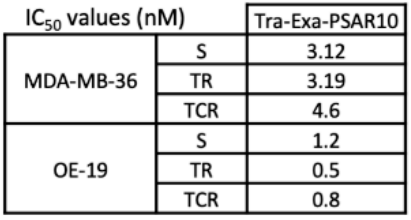

C

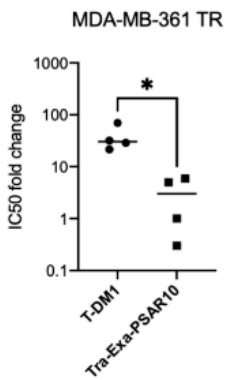

MDA-MB-361 TCR

OE-19 TR

OE-19 TCR
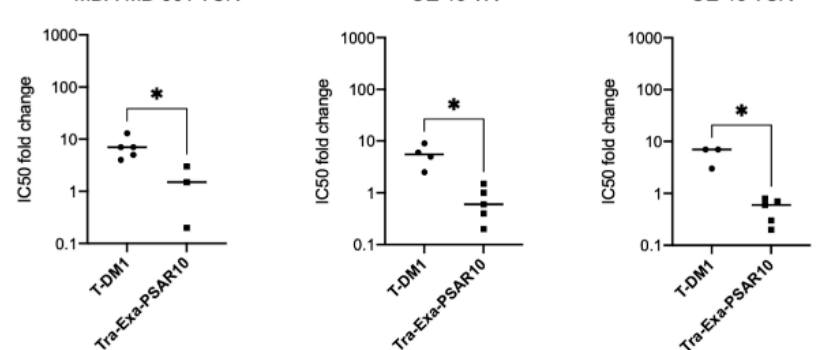

Figure 6. In vitro cytotoxicity of Tra-Exa-PSAR10 in cells resistant to T-DM1. (A) Cytotoxicity assay of T-DM1 in MDA-MB361 and OE-19 sensitive (S) and T-DM1-resistant (TR and TCR) cells showing an increase in the IC $_{50}$ values of resistant cells compared to parental. (B) Exposure of cells resistant to T-DM1 to Tra-Exa-PSAR10 showing comparable cytotoxicity in parental and T-DM1 resistant cells. (C) Relative resistance of TR and TCR cell lines to T-DM1 or Tra-Exa-PSAR10 represented as the $\mathrm{IC}_{50}$ of resistant cell line over the parental cell line for each experiment. ${ }^{*}: p<0,05$. S: sensitive cells, TR: T-DM1 resistant cells and TCR: T-DM1 resistant cells generated in the presence of ciclosporin A.

Subsequently, the cytotoxicity of Tra-Exa-PSAR10 ADC was evaluated in these cell lines (Figure 6B,C). When exposed to Tra-Exa-PSAR10, the $\mathrm{IC}_{50}$ values of sensitive, TR, and TCR cells were, respectively, $3.1 \mathrm{nM}, 3.2 \mathrm{nM}$, and $4.6 \mathrm{nM}$ in the MDA-MB-361 models and $1.2 \mathrm{nM}, 0.5 \mathrm{nM}$, and $0.8 \mathrm{nM}$ in the OE-19 models. In accordance with previous studies [33,37], these results show that resistance mechanism to T-DM1 can be circumvented and that a comparable level of sensitivity between parental cells and T-DM1-resistant cells can be obtained with an ADC on the basis of a payload having a different mechanism of action.

\section{Discussion}

Most of the ADCs currently approved or in clinical trials are conjugated to microtubule inhibitors or DNA-alkylating agents, with a low-to-moderate DAR value. The recent approval of two DAR 8 ADCs containing topoisomerase I inhibitors (DS-8201a and IMMU132) has enhanced the interest in the development of ADCs conjugated with this family of cytotoxic agents, which are 10- to 100-fold relatively less potent than the aforementioned payloads. One of the main challenges in the design of ADCs based on moderately potent payloads is thus to increase the DAR without disturbing antibody's biophysical properties. 
The design of DS-8201a was based on a novel DXd payload and an optimized linker technology that integrates a quadripeptide cleavable unit coupled with a self-immolative amino methylene spacer moiety, allowing the DAR value to be increased to 8 [23]. In this context we produced a DAR 8 ADC based on exatecan, an alternative potent topoisomerase inhibitor, using a monodisperse polysarcosine-based hydrophobicity masking drug-linker technology, which we previously applied to MMAE [29]. This drug-linker has been found to be stable in rat plasma and displays a stronger bystander killing potential when compared to DS-8201a in vitro.

The physicochemical and in vitro characterization of Tra-Exa-PSAR10 compared to naked trastuzumab, the negative control Tra-Exa-PSAR0 lacking polysarcosine, and DS8201a confirmed that neither the DAR 8 conjugation nor the incorporation of the PSAR entity affected the HER2-binding properties or the cytotoxicity of the ADCs. These data suggest that the size and the orthogonal attachment of the hydrophobicity masking entity does not negatively affect antibody binding, cell internalization, intracellular trafficking, endosomal linker cleavage, and active metabolite release. Exatecan- and DXd-based ADCs exhibited a potent and identical cytotoxicity in the low nanomolar range against breast and gastric cancer cell lines, both conjugates being compared at their identical DAR value of 8 .

Numerous studies have demonstrated that increasing the DAR value causes an increase in the overall hydrophobicity of the ADC, which induces formation of aggregates, accelerated plasma clearance, off-target toxicity, and reduced anti-tumor activity [10,12-14,35]. The recent development of hydrophobicity masking-based ADC drug-linkers, such as PEGincorporating linkers or polymeric polyacetal-based linkers, have demonstrated an efficient reduction of ADC overall hydrophobicity [14,29-32,34,53,54]. These approaches yielded maintained PK profiles and strong anti-tumor activities despite high DAR values (DAR 8-15) [31]. In our study, the PSAR incorporation in the drug-linker allowed us to generate a homogeneous DAR 8 ADC exhibiting a close-to-native-antibody hydrophobicity level as observed in hydrophobic interaction chromatography (HIC). The negative control ADC lacking PSAR surprisingly appeared almost as hydrophilic with this method, whereas DS-8201a showed a noticeable shift in retention time, indicating a higher hydrophobicity level. In vivo, all these ADCs exhibited a low clearance rate PK profile mimicking that of the native protein, except for the Tra-Exa-PSAR0 ADC. We observed that despite an apparently favorable HIC hydrophobicity level, this negative control ADC suffered from accelerated plasma clearance. The sterically hindered primary amine position of exatecan could be held responsible for this arguably unexpected result, as it has been reported that the lack of spacing at this critical position strongly influenced aggregation rates of the resulting ADCs [42]. This result suggests that designing an ADC drug-linker on the basis of an exatecan payload is not straightforward, and that simply combining exatecan with one of the most hydrophilic cleavable drug-linker unit that has been reported to date ( $\beta$-glucuronide-based drug-linker) does not seem sufficient to ensure satisfactory in vivo properties.

DS-8201a showed a favorable rat PK profile despite its relatively high overall hydrophobicity level. This observation is in accordance with previously reported primate PK studies of this ADC, even though a comparison with the native antibody was not provided [39,55]. A possible explanation could be that the PK profile of DS-8201a is favored by the progressive deruxtecan drug-linker deconjugation in plasma over time. As the DAR of the ADC decreases during the first 1-5 days of incubation in plasma (by retro-Michael deconjugation of the maleimide moiety), the overall hydrophobicity of the ADC also decreases, leading to lower ADC clearance values. Additionally, the ingenious chemical optimizations of the deruxtecan drug-linker (self-immolative amino methylene and glycolic acid spacers directly connected to the hindered primary amine of exatecan) may largely contribute to a reduced steric hindrance and thus play a significant role in the outstanding pharmacological properties of DS-8201a [40,42].

Tra-Exa-PSAR10 and DS-8201a did not show any apparent systemic toxicity in mice at $100 \mathrm{mg} / \mathrm{kg}$, a dose that is much greater than the dose inducing complete remissions in the 
HER2+xenograft models. It should be noted that the PSAR-lacking ADC Tra-Exa-PSAR0 did not induce apparent toxicity either at the same dose. At this stage and on the basis of this very exploratory toxicity study, we were not able to discriminate these three ADCs in terms of toxicity profiles or maximum tolerated doses (MTD). Comprehensive toxicological studies on PSAR-based ADCs are currently ongoing and will be reported elsewhere.

Tra-Exa-PSAR10 displayed potent anti-tumor efficacies in both breast and gastric cancer xenografts. Unsurprisingly and driven by its unfavorable PK profile, negative control ADC Tra-Exa-PSAR0 showed reduced efficacy. DS-8201a exhibited a lower antitumor activity than Tra-Exa-PSAR10 in the NCI-N87 gastric cancer model, despite having the same in vitro activity and PK profile. This result could be explained by the premature maleimide deconjugation of DS-8201a in plasma and by a lower bystander killing effect of the DXd payload compared to exatecan. This discrepancy in activity could also be driven by the difference on drug-linker cleavage strategy and kinetics (cathepsin sensitive quadripeptide versus glucuronidase-sensitive trigger) that are dependent on intracellular proteases and glucuronidases levels, respectively. In addition, the presence of extracellular glucuronidase in the tumor microenvironment reported in several studies $[45,56,57]$ could tilt the balance in favor of Tra-Exa-PSAR10.

In the ADC field, the bystander effect is characterized by the ability of the final active metabolite to passively cross cell membranes and internalize into neighboring lowantigen-expressing cells within the targeted tumor [58]. As an example, the FDA-approved trastuzumab emtansine (comprised of the $\mathrm{mAb}$ trastuzumab and the anti-microtubule agent DM1 linked through a non-cleavable SMCC linker) is devoid of such a bystander effect, as the final active metabolite is the highly polar entity Lys-SMCC-DM1, which is not capable of passive membrane diffusion [59]. In contrast, the FDA-approved DS-8201a is based on a cleavable drug-linker releasing the final metabolite DXd, which is capable of passive membrane diffusion and therefore bystander killing [37]. We herein evaluated the bystander killing potency of Tra-Exa-PSAR10 and DS-8201a in a co-culture experiment of HER2+ and HER2-cells. This experiment revealed a comparable yet stronger bystander killing effect for the exatecan-based ADC. These data encourage the development of exatecan-based ADCs for the treatment of tumors with heterogeneous antigen expression, as this payload is able to passively diffuse into neighboring antigen-negative cancer cells [58,60]. We suggest that the slight difference observed in bystander effect potency between these ADCs is driven by the slight difference in passive membrane diffusion of the two closely related camptothecin derivatives, as we observed a higher permeability coefficient (Pe) for exatecan compared to DXd in a PAMPA experiment. This result may appear counterintuitive, as it could be expected that the allegedly positively charged primary amine of exatecan (not present in the DXd payload) would negatively affect passive membrane permeability. However numerous reports provide evidence that positively charged compounds can have high membrane permeabilities, and that predicting Pe of such compounds is not obvious [61,62]. Of note it is interesting to observe that the "first generation" DXd payload, which also bears a positively charged alkyl primary amine (4-aminobutanoic acid derivative of exatecan), was devoid of passive permeability capabilities [36].

Most of the ADCs that are currently approved or under clinical investigation are based on microtubule- and DNA-targeting agents. One of the main purposes of differentiated ADC payloads is to efficiently treat tumors that become resistant to these chemotherapeutic agents or resistant to the ADC per se $[33,37,63,64]$. To address this issue, we investigated the in vitro cytotoxicity of Tra-Exa-PSAR10 in HER2+ breast (MDA-MB-361) and esophageal (OE-19) cancer cell lines that were rendered resistant to T-DM1. The characterization of T-DM1 resistance mechanisms of these two models demonstrated no alteration of HER2 expression in resistant OE-19 cells, whereas resistant MDA-MB-361 cells demonstrated heterogeneity in HER2 expression when compared to the parental cell line $[55,56]$. Our results showed that Tra-Exa-PSAR10 circumvented T-DM1-resistance in both cancer models. These results suggest that exatecan-based ADCs could be clinically useful for the treatment 
of tumors that are resistant to maytansine-based therapies, including when the target is expressed heterogeneously.

In conclusion, we herein report an innovative highly conjugated (DAR 8) ADC based on the topoisomerase I inhibitor payload exatecan. This could be achieved while preserving favorable physicochemical and pharmacological properties of the conjugate, thanks to the use of a polysarcosine-based hydrophobicity masking entity. Tra-Exa-PSAR10 showed remarkable anti-tumor properties, outperforming the FDA-approved ADC DS-8201a in a gastric cancer model. In addition, this ADC demonstrated a strong bystander killing effect as well as potent activity in T-DM1-resistant cells, suggesting a potential broad anti-tumor activity in resistant and heterogeneous tumors.

\section{Material and Methods}

\subsection{Drug-Linker Synthesis}

Chemical preparation of drug-linkers used in the present study is described in the Supplementary Material Section of the present article.

\subsection{Preparation of Antibody-Drug-Conjugates}

A solution of trastuzumab (10 mg/mL in PBS (pH 7.4) + 1 mM EDTA-Herceptin IV from Roche) was treated with 12 molar equivalent of tris(2-carboxyethyl)phosphine (TCEP) for $2 \mathrm{~h}$ at $37^{\circ} \mathrm{C}$. The fully reduced antibody was buffer-exchanged with potassium phosphate $100 \mathrm{mM}$ (pH 7.4) + $1 \mathrm{mM}$ EDTA by 3 rounds of dilution/centrifugation using an Amicon $30 \mathrm{~K}$ centrifugal filters device (Merck). Ten molar equivalents of drug-linker (from a $12 \mathrm{mM}$ DMSO stock solution) were added to the reduced antibody, while keeping residual DMSO concentration below $10 \%(v / v)$. The solution was incubated for $30 \mathrm{~min}$ at room temperature. The conjugates were buffer-exchanged/purified with PBS ( $\mathrm{pH}$ 8.0) using an Amicon $30 \mathrm{~K}$ centrifugal filters device and were incubated at $37^{\circ} \mathrm{C}$ for $24 \mathrm{~h}$ to promote complete hydrolysis of the succinimidyl moiety. The conjugates were buffer-exchanged again with PBS (pH 7.4) using an Amicon 30K centrifugal filters device and were sterile-filtered (0.2 $\mu \mathrm{M}$ PES filters). Final protein concentration was assessed spectrophotometrically at $280 \mathrm{~nm}$ using a Colibri microvolume spectrometer device (Titertek Berthold).

\subsection{Characterization of Antibody-Drug Conjugates}

Denaturing reversed phase chromatography-mass spectrometry (RPLC-MS) was performed on a Thermo UltiMate 3000 UHPLC system + Bruker Impact II Q-ToF mass spectrometer. Mobile phase A was water $+0.1 \%$ formic acid and mobile phase B was acetonitrile $+0.1 \%$ formic acid. Column was an Agilent PLRP-S $1000 \AA 2.1 \times 150 \mathrm{~mm}$ $8 \mu \mathrm{m}\left(80{ }^{\circ} \mathrm{C}\right)$. Gradient was $20 \% \mathrm{~B}$ to $50 \% \mathrm{~B}$ in $25 \mathrm{~min}$. Flow rate was $0.4 \mathrm{~mL} / \mathrm{min}$. UV detection was monitored at $280 \mathrm{~nm}$. The Q-ToF mass spectrometer was used in the $\mathrm{m} / \mathrm{z}$ range 500-3500 (ESI+). Data were deconvoluted using the MaxEnt algorithm included in the Bruker Compass software. mAb or ADC samples were diluted with $\mathrm{H}_{2} \mathrm{O}$ for injection (approximately $1.5 \mathrm{mg} / \mathrm{mL}$ final ADC concentration).

Hydrophobic interaction chromatography (HIC) was performed on an Agilent 1100 HPLC system. Column was a Tosoh TSK-GEL BUTYL-NPR $4.6 \times 35 \mathrm{~mm} 2.5 \mu \mathrm{m}\left(25^{\circ} \mathrm{C}\right)$. Mobile phase A was $1.5 \mathrm{M}\left(\mathrm{NH}_{4}\right)_{2} \mathrm{SO}_{4}+25 \mathrm{mM}$ potassium phosphate $(\mathrm{pH}$ 7.0). Mobile phase B was $25 \mathrm{mM}$ potassium phosphate $(\mathrm{pH} 7.0)+15 \%$ isopropanol $(v / v)$. Linear gradient was $0 \% \mathrm{~B}$ to $100 \% \mathrm{~B}$ in $10 \mathrm{~min}$, followed by a $3 \mathrm{~min}$ hold at $100 \% \mathrm{~B}$. Flow rate was $0.75 \mathrm{~mL} / \mathrm{min}$. UV detection was monitored at 220 and $280 \mathrm{~nm}$.

Size exclusion chromatography (SEC) was performed on an Agilent 1100 HPLC system having an extra-column volume below $15 \mu \mathrm{L}$ (equipped with short sections of $0.12 \mathrm{~mm}$ internal diameter peek tubing and a micro-volume UV flow cell). Column was an Agilent AdvanceBioSEC $300 \AA ̊ .6 \times 150 \mathrm{~mm} 2.7 \mu \mathrm{m}$ (maintained at $30^{\circ} \mathrm{C}$ ). Mobile phase was $100 \mathrm{mM}$ sodium phosphate and $200 \mathrm{mM}$ sodium chloride (pH 6.8). We added 10\% acetonitrile $(v / v)$ to the mobile phase to minimize secondary hydrophobic interactions 
with the stationary phase and prevent bacterial growth. Flow rate was $0.35 \mathrm{~mL} / \mathrm{min}$. UV detection was monitored at 220 and $280 \mathrm{~nm}$.

\subsection{HER2-Binding ELISA Affinity Assay}

Sandwich ELISA assays were performed using 96-well high-binding ELISA plates (Corning Inc., New York, NY, USA, Cat\#3590). Plates were coated using $100 \mu \mathrm{L} /$ well of the tested monoclonal antibody or ADC in PBS (pH 7.4) at $5 \mu \mathrm{g} / \mathrm{mL}$ and incubated overnight at $4{ }^{\circ} \mathrm{C}$. After 2 washes with PBS-T (PBS $+0.05 \%$ Tween-20), the plates were blocked with $200 \mu \mathrm{L} /$ well of incubation buffer (PBS-T $+0.1 \%$ BSA) for $1 \mathrm{~h}$ at room temperature. The plates were washed 4 times with PBS-T, and $100 \mu \mathrm{L}$ of a threefold dilution series of His-tagged HER2 recombinant protein (Sino Biological Inc. Cat\#10004-H08H) prepared in incubation buffer was added; then, the plates were incubated for $2 \mathrm{~h}$ at room temperature in the dark. After 5 washes with PBS-T, plates were incubated $1 \mathrm{~h}$ at room temperature with $100 \mu \mathrm{L} /$ well of HRP-conjugated anti-His Tag secondary antibody (Takara Inc. cat\#631210) diluted 1:5000 in incubation buffer. After 5 washes with PBS-T, TMB substrate solution (Thermo-Fisher cat\#N301) was added. Peroxidase activity was stopped with $0.18 \mathrm{M} \mathrm{H}_{2} \mathrm{SO}_{4}$ and absorbance was read at $450 \mathrm{~nm}$ (reference wavelength $650 \mathrm{~nm}$ ) using a Thermo Scientific MultiSkan EX microplate reader. Sigmoidal fittings were performed using GraphPad Prism 9 software.

\subsection{Ex Vivo Plasma Stability Assays}

ADC samples ( $>5 \mathrm{mg} / \mathrm{mL}$ solutions in PBS) were diluted with pure sterile SpragueDawley rat plasma (GeneTex Cat\#GTX73218) in centrifuge tubes with screw cap to yield a final ADC concentration of $400 \mu \mathrm{g} / \mathrm{mL}$ (residual PBS volume below $10 \% v / v$ ). Samples were incubated at $37^{\circ} \mathrm{C}$ and aliquots were taken at time points of $5 \mathrm{~min}, 1$ day, 2 days, 4 days, and 7 days (aliquots were kept frozen at $-80{ }^{\circ} \mathrm{C}$ until analysis). ADCs were recovered from plasma by immunocapture using AbraMag ${ }^{\mathrm{TM}}$ anti-Human magnetic beads (Eurofins Technologies Cat\#544061) following the manufacturer's protocol. Bound conjugates were extensively washed with TBS-T (TBS $+0.05 \%$ Tween-20), eluted with $0.1 \mathrm{M}$ glycine buffer ( $\mathrm{pH}$ 2.0), and neutralized with 1M TBS ( $\mathrm{pH}$ 8.0). Each sample was analyzed by denaturing reversed phase chromatography-mass spectrometry as described above.

\subsection{Cell Culture}

Human breast adenocarcinoma cell lines MDA-MB-361, MDA-MB-453, and SKBR3 were cultured in DMEM supplemented with $10 \%$ fetal calf serum and $100 \mu \mathrm{g} / \mathrm{mL}$ streptomycin at $37{ }^{\circ} \mathrm{C}$ and incubated under a $5 \% \mathrm{CO}_{2}$ atmosphere. The human breast adenocarcinoma BT-474, the gastric cancer NCI-N87, and the esophageal OE-19 cancer cell lines were cultured in RPMI medium supplemented with $10 \%$ fetal calf serum and $100 \mathrm{\mu g} / \mathrm{mL}$ streptomycin at $37^{\circ} \mathrm{C}$ and incubated under a $5 \% \mathrm{CO}_{2}$ atmosphere.

MDA-MB-361 and OE-19 cells resistant to T-DM1 were cultured in DMEM and RPMI complete mediums, respectively, plus $0.4 \mathrm{nM}$ of T-DM1, with (TR) or without (TCR) $1 \mu \mathrm{g} / \mathrm{mL}$ of ciclosporin A (Cat\#C3662, Sigma-Aldrich, St. Louis, MI, USA).

\subsection{Flow Cytometry}

For HER2 cell surface quantification, cells were incubated for $30 \mathrm{~min}$ with antiHER2 APC-conjugated antibody (BD Bioscience, Franklin Lakes, NJ, USA, Cat\#340554) or mouse IgG1k control isotype conjugated to APC (BD Pharmingen, San Diego, CA, USA, Cat\#555751). For mAb or ADC cell binding, the tested compound was conjugated to APC fluorochrome using LYNX Rapid APC Antibody Conjugation Kit according to the manufacturer's protocol (Bio-Rad, Hercules, CA, USA, Cat\#LNK032APC). Analysis was performed using a BD Fortessa flow cytometer controlled by BD FACSDiva software (BD Biosciences) and data were analyzed using FlowJo software (BD Bioscience). 


\subsection{In Vitro Cytotoxicity Assays}

In vitro cytotoxicity of conjugates was assessed on several antigen-positive cell lines. Cells were plated in 96-well plates at an appropriate density depending on the cell line (between 1000 and 10,000 cells per well in $100 \mu \mathrm{L}$ of appropriate culture media) and incubated at $37^{\circ} \mathrm{C}$ for $24 \mathrm{~h}$. Serial dilutions of the tested compound previously dissolved in culture media were added, and incubation was carried out at $37^{\circ} \mathrm{C}$ for $144 \mathrm{~h}$. MTT $(5 \mathrm{mg} / \mathrm{mL}, 20 \mu \mathrm{L}$, Sigma-Aldrich) was added into the wells, and incubation was continued for 2 to $4 \mathrm{~h}$ at $37^{\circ} \mathrm{C}$. Culture media was then carefully removed, and well content was homogeneously dissolved with $0.1 \mathrm{~N} \mathrm{HCl}$ /isopropanol. Absorbance values were measured on a Thermo Scientific Multiskan EX microplate reader using a wavelength of $570 \mathrm{~nm}$ (with a reference wavelength of $690 \mathrm{~nm}$ ). The $\mathrm{IC}_{50}$ concentration values compared to untreated control cells were determined using inhibition dose response curve fitting (GraphPad Prism 9).

\subsection{PAMPA Permeability Assay}

Passive permeabilities of exatecan mesylate and DXd (both from MedChemExpress) were compared by parallel artificial membrane permeability assay (PAMPA assay) using a GenTest ${ }^{\mathrm{TM}}$ pre-coated PAMPA plate system (Corning, New York, NY, USA, Cat\#353015), following the manufacturer's protocol and calculation procedures. The assay buffer was PBS $(\mathrm{pH} 7.4)+10 \% \mathrm{MeOH}(v / v)$. The initial compound concentration in the donor compartment was $100 \mu \mathrm{M}$. Residual DMSO in the assay was kept below $1 \%(v / v)$. The permeability partitioning was realized for $5 \mathrm{~h}$ at room temperature while stirring the plate at $300 \mathrm{rpm}$ on a Heidolph Titramax 101 device. Final concentration of tested compound in the donor and acceptor compartments was assessed by HPLC-UV, against known calibration curves of compounds.

\subsection{In Vitro Bystander Killing Assay}

SKBR-3 and A549 cells were seeded in single culture or co-culture in 96-well plates at a density of 8000 cells per well and incubated overnight at $37{ }^{\circ} \mathrm{C}$ under a $5 \% \mathrm{CO}_{2}$ atmosphere. The incubation media was removed and $100 \mu \mathrm{L}$ of a $10 \mathrm{nM}$ solution of tested conjugate in DMEM complete medium were added to the plate and incubated for 5 days. Cells were then collected and transferred into a round-bottom 96-well plate suitable for high-throughput flow cytometry. Cells were rinsed 3 times with PBS, resuspended in $50 \mu \mathrm{L}$ DPBS, and incubated for $30 \mathrm{~min}$ at room temperature in the dark with anti-HER2 APC-conjugated antibody (BD Bioscience, Cat\#340554) and eBioscience Fixable Viability Dye eFluor 780 (Thermo Scientific, Waltham, MA, USA, Cat\#650865-14). Flow cytometry analysis was performed using a BD Fortessa flow cytometer controlled by BD FACSDiva software (BD Biosciences), and data were analyzed using FlowJo software (BD Bioscience).

\subsection{In Vivo Studies}

All animal procedures were performed in accordance with the European Union directive 86/609/EEC. Experiments were performed under individual permit and in animal care facilities accredited by the French Ministry of Agriculture. The study was approved by the local animal ethics committee (CECCAPP). The rat PK and NCI-N87 xenograft studies were outsourced to the contract research organization (CRO) Antinéo (www.antineo.fr; accessed on 10 August 2020).

\subsection{Rat PK Study}

ADCs were injected at $3 \mathrm{mg} / \mathrm{kg}$ in female Sprague-Dawley rats (4-6 weeks oldCharles River) via the tail vein ( 3 animals per group, randomly assigned). Blood was drawn into citrate tubes via retro-orbital bleeding at $10 \mathrm{~min}, 4 \mathrm{~h}, 1$ day, 2 days, 4 days, 7 days, 14 days, and 21 days; processed to plasma; and stored at $-80{ }^{\circ} \mathrm{C}$ until analysis. ADC concentration was assessed using a human IgG ELISA kit (Stemcell Technologies, Cambridge, MA, USA, Cat\#01994) according to the manufacturer's protocol. Standard 
curves of trastuzumab antibody were used for quantification. Pharmacokinetic parameters (clearance, half-life, and AUC) were calculated by two-compartmental analysis using Microsoft Excel software incorporating PK functions (add-in developed by Usansky et al., Department of Pharmacokinetics and Drug Metabolism, Allergan, Irvine, CA, USA).

\subsection{In Vivo Efficacy Experiments}

In vivo efficacy studies were conducted in severe combined immunodeficiency (SCID) mice $(n=6)$. We suspended $5 \times 10^{6}$ BT- 474 or NCI-N87 cells in $0.2 \mathrm{~mL}$ of PBS (pH 7.4) and injected them subcutaneously in the left flank of mice. For the breast cancer BT-474 xenograft experiment, treatment was initiated when the tumor volume averaged $150 \mathrm{~mm}^{3}$ and was administered once intravenously at an ADC concentration of $10 \mathrm{mg} / \mathrm{kg}(n=6$ mice per group). For the gastric cancer NCI-N87 xenograft experiment, treatment was initiated when the tumor volume averaged $150 \mathrm{~mm}^{3}$ and was administered once intravenously at a sub-curative dose of $1 \mathrm{mg} / \mathrm{kg}$ ( $n=8$ mice per group). Tumor volumes were measured every 3-5 days using a caliper device (length $x$ width) and calculated using the following formula $V=4 / 3 \times \pi \times R^{3}$, where $R$ represents the radius. Mice were sacrificed when the tumor volume exceeded $1500 \mathrm{~mm}^{3}$. No significant body weight changes were observed during these studies.

\subsection{Mice Tolerability Experiments}

To assess mouse tolerability of ADCs of the present study, we treated SCID mice $(n=3)$ with a single intraperitoneal dose of $100 \mathrm{mg} / \mathrm{kg}$ of ADC compound. Mice were observed for weight loss or apparent signs of toxicity over the course of 10 days.

\subsection{Statistics}

In vitro experiments were conducted at least 3 times, and data are presented as means +/ - SD or single representative experiments. Statistical significance was evaluated using Student's $t$-test and calculated using GraphPad Prism 9 software. IC $\mathrm{C}_{50}$ 's and $\mathrm{EC}_{50}$ 's were calculated using GraphPad Prism 9 software. In vivo experiment data are represented as means +/ - SD. Statistical significance was evaluated using Mann-Whitney test and calculated using GraphPad Prism 9 software. $p$-values are represented as * $(p<0.05)$, ** $(p<0.01)$, and ${ }^{* * *}(p<0.001)$, and "ns" stands for non-significant.

Supplementary Materials: Supplementary figures and organic syntheses. The following are available online at https:/ /www.mdpi.com/1424-8247/14/3/247/s1, Figure S1: Chemical structures of Exatecan and DXd payloads, Figure S2: Structures of trastuzumab-based antibody-drug conjugates, Figure S3: Representative RPLC-QToF characterization of ADCs, Figure S4: Pharmacokinetic parameters (Sprague-Dawley rat PK study), Figure S5: Survival curve of the SCID/NCI-N87 xenograft study.

Author Contributions: L.C. and W.V. designed and performed the experiments, interpreted the results, and wrote the manuscript. E.F., A.M. and E.-L.M. performed the experiments. B.J. and G.F. designed and performed the experiments and interpreted the results. C.D. designed the experiments, interpreted the results, and wrote the manuscript. All authors have read and agreed to the published version of the manuscript.

Funding: This research was funded by Mablink Bioscience and the Cancéropole Auvergne-RhôneAlpes (CLARA).

Institutional Review Board Statement: The study was conducted according to the guidelines of the Declaration of Helsinki, and approved by the Ethics Committee of the Université de Lyon.

Informed Consent Statement: Not applicable.

Data Availability Statement: Not applicable.

Acknowledgments: L.C. is supported by the French National Association of Research and Technology (ANRT). 
Conflicts of Interest: L.C. and A.M. are employees of Mablink Bioscience. W.V., B.J. and C.D. are shareholders of Mablink Bioscience.

\section{References}

1. Beck, A.; Goetsch, L.; Dumontet, C.; Corvaïa, N. Strategies and Challenges for the next Generation of Antibody-Drug Conjugates. Nat. Rev. Drug Discov. 2017, 16, 315-337. [CrossRef]

2. Strebhardt, K.; Ullrich, A. Paul Ehrlich's Magic Bullet Concept: 100 Years of Progress. Nat. Rev. Cancer 2008, 8, 473-480. [CrossRef]

3. Keam, S.J. Trastuzumab Deruxtecan: First Approval. Drugs 2020, 80, 501-508. [CrossRef]

4. Deeks, E.D. Polatuzumab Vedotin: First Global Approval. Drugs 2019, 79, 1467-1475. [CrossRef] [PubMed]

5. Chang, E.; Weinstock, C.; Zhang, L.; Charlab, R.; Dorff, S.E.; Gong, Y.; Hsu, V.; Li, F.; Ricks, T.K.; Song, P.; et al. FDA Approval Summary: Enfortumab Vedotin for Locally Advanced or Metastatic Urothelial Carcinoma. Clin. Cancer Res. 2020. [CrossRef]

6. Syed, Y.Y. Sacituzumab Govitecan: First Approval. Drugs 2020, 80, 1019-1025. [CrossRef] [PubMed]

7. Markham, A. Belantamab Mafodotin: First Approval. Drugs 2020, 80, 1607-1613. [CrossRef] [PubMed]

8. Hafeez, U.; Parakh, S.; Gan, H.K.; Scott, A.M. Antibody-Drug Conjugates for Cancer Therapy. Molecules 2020, 25, 4764. [CrossRef] [PubMed]

9. Joubert, N.; Beck, A.; Dumontet, C.; Denevault-Sabourin, C. Antibody-Drug Conjugates: The Last Decade. Pharmaceuticals 2020, 13, 245. [CrossRef]

10. Buecheler, J.W.; Winzer, M.; Tonillo, J.; Weber, C.; Gieseler, H. Impact of Payload Hydrophobicity on the Stability of Antibody-Drug Conjugates. Mol. Pharm. 2018, 15, 2656-2664. [CrossRef]

11. Ratanji, K.D.; Derrick, J.P.; Dearman, R.J.; Kimber, I. Immunogenicity of Therapeutic Proteins: Influence of Aggregation. J. Immunotoxicol. 2014, 11, 99-109. [CrossRef]

12. Lyon, R.P.; Bovee, T.D.; Doronina, S.O.; Burke, P.J.; Hunter, J.H.; Neff-LaFord, H.D.; Jonas, M.; Anderson, M.E.; Setter, J.R.; Senter, P.D. Reducing Hydrophobicity of Homogeneous Antibody-Drug Conjugates Improves Pharmacokinetics and Therapeutic Index. Nat. Biotechnol. 2015, 33, 733-735. [CrossRef]

13. Hamblett, K.J.; Senter, P.D.; Chace, D.F.; Sun, M.M.C.; Lenox, J.; Cerveny, C.G.; Kissler, K.M.; Bernhardt, S.X.; Kopcha, A.K.; Zabinski, R.F.; et al. Effects of Drug Loading on the Antitumor Activity of a Monoclonal Antibody Drug Conjugate. Clin. Cancer Res. 2004, 10, 7063-7070. [CrossRef]

14. Simmons, J.K.; Burke, P.J.; Cochran, J.H.; Pittman, P.G.; Lyon, R.P. Reducing the Antigen-Independent Toxicity of Antibody-Drug Conjugates by Minimizing Their Non-Specific Clearance through PEGylation. Toxicol. Appl. Pharm. 2020, 392, 114932. [CrossRef]

15. Teicher, B.A.; Chari, R.V.J. Antibody Conjugate Therapeutics: Challenges and Potential. Clin. Cancer Res. 2011, $17,6389-6397$. [CrossRef]

16. Diamantis, N.; Banerji, U. Antibody-Drug Conjugates-an Emerging Class of Cancer Treatment. Br. J. Cancer 2016, 114, 362-367. [CrossRef]

17. Jeffrey, S.C.; Burke, P.J.; Lyon, R.P.; Meyer, D.W.; Sussman, D.; Anderson, M.; Hunter, J.H.; Leiske, C.I.; Miyamoto, J.B.; Nicholas, N.D.; et al. A Potent Anti-CD70 Antibody-Drug Conjugate Combining a Dimeric Pyrrolobenzodiazepine Drug with Site-Specific Conjugation Technology. Bioconjugate Chem. 2013, 24, 1256-1263. [CrossRef]

18. Mantaj, J.; Jackson, P.J.M.; Rahman, K.M.; Thurston, D.E. From Anthramycin to Pyrrolobenzodiazepine (PBD)-Containing Antibody-Drug Conjugates (ADCs). Angew. Chem. Int. Ed. 2017, 56, 462-488. [CrossRef]

19. Walsh, S.J.; Bargh, J.D.; Dannheim, F.M.; Hanby, A.R.; Seki, H.; Counsell, A.J.; Ou, X.; Fowler, E.; Ashman, N.; Takada, Y.; et al. Site-Selective Modification Strategies in Antibody-Drug Conjugates. Chem. Soc. Rev. 2021, 50, 1305-1353. [CrossRef]

20. Harper, J.; Lloyd, C.; Dimasi, N.; Toader, D.; Marwood, R.; Lewis, L.; Bannister, D.; Jovanovic, J.; Fleming, R.; D’Hooge, F.; et al. Preclinical Evaluation of MEDI0641, a Pyrrolobenzodiazepine-Conjugated Antibody-Drug Conjugate Targeting 5T4. Mol. Cancer Ther. 2017, 16, 1576-1587. [CrossRef]

21. Saber, H.; Simpson, N.; Ricks, T.K.; Leighton, J.K. An FDA Oncology Analysis of Toxicities Associated with PBD-Containing Antibody-Drug Conjugates. Regul. Toxicol. Pharmacol. 2019, 107, 104429. [CrossRef] [PubMed]

22. Hartley, J.A. Antibody-Drug Conjugates (ADCs) Delivering Pyrrolobenzodiazepine (PBD) Dimers for Cancer Therapy. Expert Opin. Biol. Ther. 2020, 1-13. [CrossRef]

23. Ogitani, Y.; Aida, T.; Hagihara, K.; Yamaguchi, J.; Ishii, C.; Harada, N.; Soma, M.; Okamoto, H.; Oitate, M.; Arakawa, S.; et al. DS-8201a, A Novel HER2-Targeting ADC with a Novel DNA Topoisomerase I Inhibitor, Demonstrates a Promising Antitumor Efficacy with Differentiation from T-DM1. Clin. Cancer Res. 2016, 22, 5097-5108. [CrossRef]

24. Goldenberg, D.M.; Cardillo, T.M.; Govindan, S.V.; Rossi, E.A.; Sharkey, R.M. Trop-2 Is a Novel Target for Solid Cancer Therapy with Sacituzumab Govitecan (IMMU-132), an Antibody-Drug Conjugate (ADC). Oncotarget 2015, 6, 22496-22512. [CrossRef]

25. Pommier, Y.; Leo, E.; Zhang, H.; Marchand, C. DNA Topoisomerases and Their Poisoning by Anticancer and Antibacterial Drugs. Chem. Biol. 2010, 17, 421-433. [CrossRef]

26. Pommier, Y. Topoisomerase I Inhibitors: Camptothecins and Beyond. Nat. Rev. Cancer 2006, 6, 789-802. [CrossRef]

27. Zunino, F.; Pratesi, G. Camptothecins in Clinical Development. Expert Opin. Investig. Drugs 2004, 13, 269-284. [CrossRef] [PubMed]

28. Bailly, C. Irinotecan: 25 Years of Cancer Treatment. Pharm. Res. 2019, 148, 104398. [CrossRef] 
29. Viricel, W.; Fournet, G.; Beaumel, S.; Perrial, E.; Papot, S.; Dumontet, C.; Joseph, B. Monodisperse Polysarcosine-Based HighlyLoaded Antibody-Drug Conjugates. Chem. Sci. 2019, 10, 4048-4053. [CrossRef]

30. Burke, P.J.; Hamilton, J.Z.; Jeffrey, S.C.; Hunter, J.H.; Doronina, S.O.; Okeley, N.M.; Miyamoto, J.B.; Anderson, M.E.; Stone, I.J.; Ulrich, M.L.; et al. Optimization of a PEGylated Glucuronide-Monomethylauristatin E Linker for Antibody-Drug Conjugates. Mol. Cancer Ther. 2017, 16, 116-123. [CrossRef]

31. Yurkovetskiy, A.V.; Yin, M.; Bodyak, N.; Stevenson, C.A.; Thomas, J.D.; Hammond, C.E.; Qin, L.; Zhu, B.; Gumerov, D.R.; Ter-Ovanesyan, E.; et al. A Polymer-Based Antibody-Vinca Drug Conjugate Platform: Characterization and Preclinical Efficacy. Cancer Res. 2015, 75, 3365-3372. [CrossRef]

32. Shao, S.; Tsai, M.-H.; Lu, J.; Yu, T.; Jin, J.; Xiao, D.; Jiang, H.; Han, M.; Wang, M.; Wang, J. Site-Specific and Hydrophilic ADCs through Disulfide-Bridged Linker and Branched PEG. Bioorg. Med. Chem. Lett. 2018, 28, 1363-1370. [CrossRef]

33. Le Joncour, V.; Martins, A.; Puhka, M.; Isola, J.; Salmikangas, M.; Laakkonen, P.; Joensuu, H.; Barok, M. A Novel Anti-HER2 Antibody-Drug Conjugate XMT-1522 for HER2-Positive Breast and Gastric Cancers Resistant to Trastuzumab Emtansine. Mol. Cancer Ther. 2019, 18, 1721-1730. [CrossRef]

34. Shao, T.; Chen, T.; Chen, Y.; Liu, X.; Chen, Y.-L.; Wang, Q.; Zhu, T.; Guo, M.; Li, H.; Ju, D.; et al. Construction of Paclitaxel-Based Antibody-Drug Conjugates with a PEGylated Linker to Achieve Superior Therapeutic Index. Signal. Transduct. Target. Ther. 2020, 5, 132. [CrossRef]

35. Meyer, D.W.; Bou, L.B.; Shum, S.; Jonas, M.; Anderson, M.E.; Hamilton, J.Z.; Hunter, J.H.; Wo, S.W.; Wong, A.O.; Okeley, N.M.; et al. An in Vitro Assay Using Cultured Kupffer Cells Can Predict the Impact of Drug Conjugation on in Vivo Antibody Pharmacokinetics. Mol. Pharm. 2020, 17, 802-809. [CrossRef]

36. Ogitani, Y.; Hagihara, K.; Oitate, M.; Naito, H.; Agatsuma, T. Bystander Killing Effect of DS-8201a, a Novel Anti-Human Epidermal Growth Factor Receptor 2 Antibody-Drug Conjugate, in Tumors with Human Epidermal Growth Factor Receptor 2 Heterogeneity. Cancer Sci. 2016, 107, 1039-1046. [CrossRef]

37. Takegawa, N.; Nonagase, Y.; Yonesaka, K.; Sakai, K.; Maenishi, O.; Ogitani, Y.; Tamura, T.; Nishio, K.; Nakagawa, K.; Tsurutani, J. DS-8201a, a New HER2-Targeting Antibody-Drug Conjugate Incorporating a Novel DNA Topoisomerase I Inhibitor, Overcomes HER2-Positive Gastric Cancer T-DM1 Resistance. Int. J. Cancer 2017, 141, 1682-1689. [CrossRef]

38. Iwata, T.N.; Ishii, C.; Ishida, S.; Ogitani, Y.; Wada, T.; Agatsuma, T. A HER2-Targeting Antibody-Drug Conjugate, Trastuzumab Deruxtecan (DS-8201a), Enhances Antitumor Immunity in a Mouse Model. Mol. Cancer Ther. 2018, 17, 1494-1503. [CrossRef]

39. Nagai, Y.; Oitate, M.; Shiozawa, H.; Ando, O. Comprehensive Preclinical Pharmacokinetic Evaluations of Trastuzumab Deruxtecan (DS-8201a), a HER2-Targeting Antibody-Drug Conjugate, in Cynomolgus Monkeys. Xenobiotica 2019, 49, 1086-1096. [CrossRef]

40. Ogitani, Y.; Abe, Y.; Iguchi, T.; Yamaguchi, J.; Terauchi, T.; Kitamura, M.; Goto, K.; Goto, M.; Oitate, M.; Yukinaga, H.; et al. Wide Application of a Novel Topoisomerase I Inhibitor-Based Drug Conjugation Technology. Bioorg. Med. Chem. Lett. 2016, 26, 5069-5072. [CrossRef]

41. Andrikopoulou, A.; Zografos, E.; Liontos, M.; Koutsoukos, K.; Dimopoulos, M.-A.; Zagouri, F. Trastuzumab Deruxtecan (DS-8201a): The Latest Research and Advances in Breast Cancer. Clin. Breast Cancer 2020. [CrossRef]

42. Nakada, T.; Masuda, T.; Naito, H.; Yoshida, M.; Ashida, S.; Morita, K.; Miyazaki, H.; Kasuya, Y.; Ogitani, Y.; Yamaguchi, J.; et al. Novel Antibody Drug Conjugates Containing Exatecan Derivative-Based Cytotoxic Payloads. Bioorg. Med. Chem. Lett. 2016, 26, 1542-1545. [CrossRef]

43. Mitsui, I.; Kumazawa, E.; Hirota, Y.; Aonuma, M.; Sugimori, M.; Ohsuki, S.; Uoto, K.; Ejima, A.; Terasawa, H.; Sato, K. A New Water-Soluble Camptothecin Derivative, DX-8951f, Exhibits Potent Antitumor Activity against Human Tumors in Vitro and in Vivo. Jpn. J. Cancer Res. 1995, 86, 776-782. [CrossRef]

44. Legigan, T.; Clarhaut, J.; Renoux, B.; Tranoy-Opalinski, I.; Monvoisin, A.; Berjeaud, J.-M.; Guilhot, F.; Papot, S. Synthesis and Antitumor Efficacy of a $\beta$-Glucuronidase-Responsive Albumin-Binding Prodrug of Doxorubicin. J. Med. Chem. 2012, 55, 4516-4520. [CrossRef]

45. Bargh, J.D.; Isidro-Llobet, A.; Parker, J.S.; Spring, D.R. Cleavable Linkers in Antibody-Drug Conjugates. Chem. Soc. Rev. 2019, 48, 4361-4374. [CrossRef]

46. Tetko, I.V.; Gasteiger, J.; Todeschini, R.; Mauri, A.; Livingstone, D.; Ertl, P.; Palyulin, V.A.; Radchenko, E.V.; Zefirov, N.S.; Makarenko, A.S.; et al. Virtual Computational Chemistry Laboratory - Design and Description. J. Comput. Aided Mol. Des. 2005, 19, 453-463. [CrossRef]

47. Christie, R.J.; Fleming, R.; Bezabeh, B.; Woods, R.; Mao, S.; Harper, J.; Joseph, A.; Wang, Q.; Xu, Z.-Q.; Wu, H.; et al. Stabilization of Cysteine-Linked Antibody Drug Conjugates with N-Aryl Maleimides. J. Control. Release 2015, 220, 660-670. [CrossRef]

48. Szijj, P.A.; Bahou, C.; Chudasama, V. Minireview: Addressing the Retro-Michael Instability of Maleimide Bioconjugates. Drug Discov. Today Technol. 2018, 30, 27-34. [CrossRef]

49. Alley, S.C.; Benjamin, D.R.; Jeffrey, S.C.; Okeley, N.M.; Meyer, D.L.; Sanderson, R.J.; Senter, P.D. Contribution of Linker Stability to the Activities of Anticancer Immunoconjugates. Bioconjugate Chem. 2008, 19, 759-765. [CrossRef]

50. Ovacik, M.; Lin, K. Tutorial on Monoclonal Antibody Pharmacokinetics and Its Considerations in Early Development. Clin. Transl. Sci. 2018, 11, 540-552. [CrossRef]

51. Sauveur, J.; Matera, E.-L.; Chettab, K.; Valet, P.; Guitton, J.; Savina, A.; Dumontet, C. Esophageal Cancer Cells Resistant to T-DM1 Display Alterations in Cell Adhesion and the Prostaglandin Pathway. Oncotarget 2018, 9, 21141-21155. [CrossRef] [PubMed] 
52. Sauveur, J.; Conilh, L.; Beaumel, S.; Chettab, K.; Jordheim, L.-P.; Matera, E.-L.; Dumontet, C. Characterization of T-DM1-Resistant Breast Cancer Cells. Pharm. Res. Perspect. 2020, 8, e00617. [CrossRef] [PubMed]

53. Hartimath, S.V.; El-Sayed, A.; Makhlouf, A.; Bernhard, W.; Gonzalez, C.; Hill, W.; Parada, A.C.; Barreto, K.; Geyer, C.R.; Fonge, H. Therapeutic Potential of Nimotuzumab PEGylated-Maytansine Antibody Drug Conjugates against EGFR Positive Xenograft. Oncotarget 2019, 10, 1031-1044. [CrossRef] [PubMed]

54. Bryden, F.; Martin, C.; Letast, S.; Lles, E.; Viéitez-Villemin, I.; Rousseau, A.; Colas, C.; Brachet-Botineau, M.; Allard-Vannier, E.; Larbouret, C.; et al. Impact of Cathepsin B-Sensitive Triggers and Hydrophilic Linkers on in Vitro Efficacy of Novel Site-Specific Antibody-Drug Conjugates. Org. Biomol. Chem. 2018, 16, 1882-1889. [CrossRef]

55. Yin, O.; Xiong, Y.; Endo, S.; Yoshihara, K.; Garimella, T.; AbuTarif, M.; Wada, R.; LaCreta, F. Population Pharmacokinetics of Trastuzumab Deruxtecan in Patients With HER2-Positive Breast Cancer and Other Solid Tumors. Clin. Pharm. Ther. 2020. [CrossRef]

56. Rivas, P.L.; Müller, C.; Breunig, C.; Hechler, T.; Pahl, A.; Arosio, D.; Belvisi, L.; Pignataro, L.; Corso, A.D.; Gennari, C. $\beta-$ Glucuronidase Triggers Extracellular MMAE Release from an Integrin-Targeted Conjugate. Org. Biomol. Chem. 2019, 17, 4705-4710. [CrossRef] [PubMed]

57. Renoux, B.; Raes, F.; Legigan, T.; Péraudeau, E.; Eddhif, B.; Poinot, P.; Tranoy-Opalinski, I.; Alsarraf, J.; Koniev, O.; Kolodych, S.; et al. Targeting the Tumour Microenvironment with an Enzyme-Responsive Drug Delivery System for the Efficient Therapy of Breast and Pancreatic Cancers. Chem. Sci. 2017, 8, 3427-3433. [CrossRef]

58. Kovtun, Y.V.; Audette, C.A.; Ye, Y.; Xie, H.; Ruberti, M.F.; Phinney, S.J.; Leece, B.A.; Chittenden, T.; Blättler, W.A.; Goldmacher, V.S. Antibody-Drug Conjugates Designed to Eradicate Tumors with Homogeneous and Heterogeneous Expression of the Target Antigen. Cancer Res. 2006, 66, 3214-3221. [CrossRef]

59. Lambert, J.M.; Chari, R.V.J. Ado-Trastuzumab Emtansine (T-DM1): An Antibody-Drug Conjugate (ADC) for HER2-Positive Breast Cancer. J. Med. Chem. 2014, 57, 6949-6964. [CrossRef]

60. Staudacher, A.H.; Brown, M.P. Antibody Drug Conjugates and Bystander Killing: Is Antigen-Dependent Internalisation Required? Br. J. Cancer 2017, 117, 1736-1742. [CrossRef]

61. Fischer, H.; Kansy, M.; Avdeef, A.; Senner, F. Permeation of Permanently Positive Charged Molecules through Artificial Membranes-Influence of Physico-Chemical Properties. Eur. J. Pharm. Sci. 2007, 31, 32-42. [CrossRef]

62. Avdeef, A.; Artursson, P.; Neuhoff, S.; Lazorova, L.; Gråsjö, J.; Tavelin, S. Caco-2 Permeability of Weakly Basic Drugs Predicted with the Double-Sink PAMPA PKaflux Method. Eur. J. Pharm. Sci. 2005, 24, 333-349. [CrossRef]

63. García-Alonso, S.; Ocaña, A.; Pandiella, A. Resistance to Antibody-Drug Conjugates. Cancer Res. 2018. [CrossRef]

64. Collins, D.M.; Bossenmaier, B.; Kollmorgen, G.; Niederfellner, G. Acquired Resistance to Antibody-Drug Conjugates. Cancers 2019, 11, 394. [CrossRef] 